\section{Tracheobronchomegaly: an unusual presentation of a rare condition}

\author{
D L Smith, N Withers, B Holloway, \\ J V Collins
}

\begin{abstract}
Tracheobronchomegaly is a rare cause of recurrent chest infections often with persistent, unproductive cough. A case is described which presented as a severe life threatening pneumonia in which the bronchoscopic, radiographic, and computed tomographic findings are given.
\end{abstract}

(Thorax 1994;49:840-841)

\section{Case history}

A 56 year old white man presented with a two week history of increasing dyspnoea and nonproductive cough. He had received erythromycin, $2 \mathrm{~g}$ daily, for a week from his general practitioner and, having failed to respond to this, had been given a second week of the same antibiotic together with prednisolone, $30 \mathrm{mg}$ daily. He had been fit and well until five years before admission when he had developed a productive cough which resolved following a short course of oral antibiotics. He had experienced six further episodes, all of which promptly resolved with oral antibiotics. Between these episodes he had no respiratory symptoms and remained an active squash player, his last game being three weeks before hospitalisation. He had not smoked for five years but had a 35 pack/year smoking history.

On examination he was febrile and significantly dyspnoeic at rest. Both lung bases were dull to percussion and there was widespread wheeze with additional coarse crackles at the right lung base. The initial chest radiograph showed bilateral lower zone consolidation with small pleural effusions. Arterial blood gas analysis whilst breathing air showed severe hypoxaemia with a $\mathrm{PaO}_{2}$ of $7.2 \mathrm{kPa}$ and $\mathrm{PaCO}_{2}$ of $4.3 \mathrm{kPa}$. The white cell count was elevated at $31.6 \times 10^{9} / 1$ with a predominant neutrophilia. A diagnosis of severe bilateral pneumonia was made and the patient was started on broad spectrum intravenous antibiotics, nebulised bronchodilators, and oxygen. The prednisolone was discontinued. Microscopy and culture of sputum yielded normal respiratory flora only. Blood cultures remained sterile and serological testing for atypical respiratory pathogens was negative.

For the next 48 hours he remained extremely unwell with persistent fever, tachycardia, and tachypnoea. Oxygenation remained poor with a $\mathrm{PaO}_{2}$ of $8.4 \mathrm{kPa}$ despite an $\mathrm{FiO}_{2}$ of $60 \%$. After 72 hours there were signs of a slow clinical recovery but a repeat chest radiograph showed wor- sening consolidation at both bases and suggested increasing pleural effusions. Surprisingly, an ultrasound scan of the chest failed to confirm the presence of significant pleural fluid collections.

In view of his slow progress a computed tomographic scan of the thorax and fibreoptic bronchoscopy with transbronchial biopsy were performed. Videobronchoscopy revealed an abnormal trachea flattened anteroposteriorly with a corrugated appearance to the anterior wall. Two cartilaginous nodules were noted anteriorly approximately $3 \mathrm{~cm}$ above the carina which were examined by biopsy (fig 1). The right and left lower lobe bronchi contained copious mucopurulent secretions which were aspirated and sent for microbiological examination. Transbronchial biopsies were taken from the right lower lobe. A computed tomographic scan of the thorax demonstrated widespread consolidation of both lower lobes with air bronchograms. In addition there was considerable dilatation of the trachea and proximal bronchial tree. The two nodules noted at bronchoscopy were demonstrated and the trachea was seen to have an unusual cross sectional profile with a lateral dimension of $5.2 \mathrm{~cm}$ (fig 2). These features were diagnostic of tracheobronchomegaly (Mounier-Kuhn syndrome). Culture of the aspirated mucopurulent bronchial secretions yielded no growth. Biopsy samples of the tracheal nodules showed cartilaginous material on histological examination and the transbronchial biopsies showed changes consistent with an organising pneumonia.

The patient made a satisfactory recovery on intravenous and subsequently oral antibiotic therapy and was discharged after 13 days. Repeat computed tomographic scans following clinical recovery demonstrated complete resolution of the bilateral lower lobe consolidation, flow-volume loops showed no evidence of large

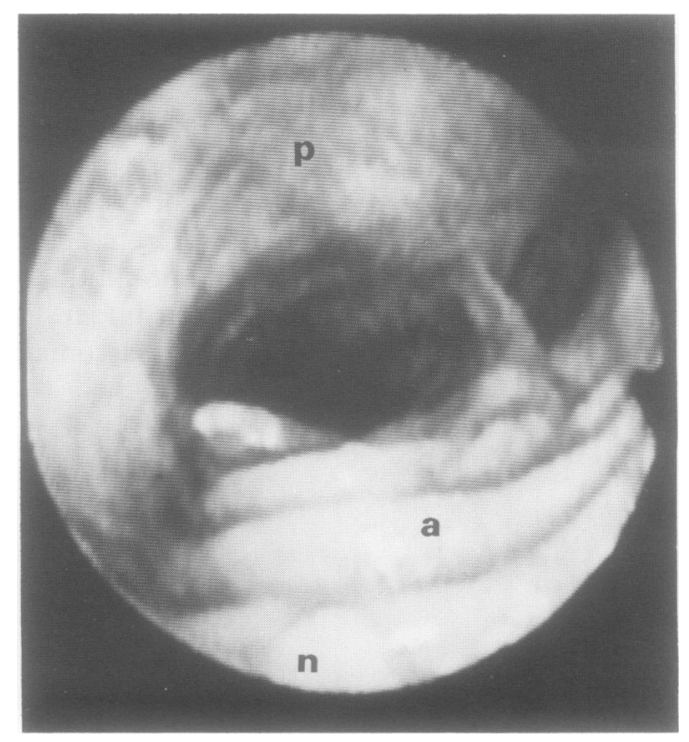

Figure 1 Videobronchoscopic appearance of trachea with a cartilaginous nodule ( $n$ ) in the foreground. The striking corrugated appearance of the anterior wall of the trachea (a) together with the abrupt change to the smooth appearance of the posterior tracheal wall ( $p)$ is well seen. 


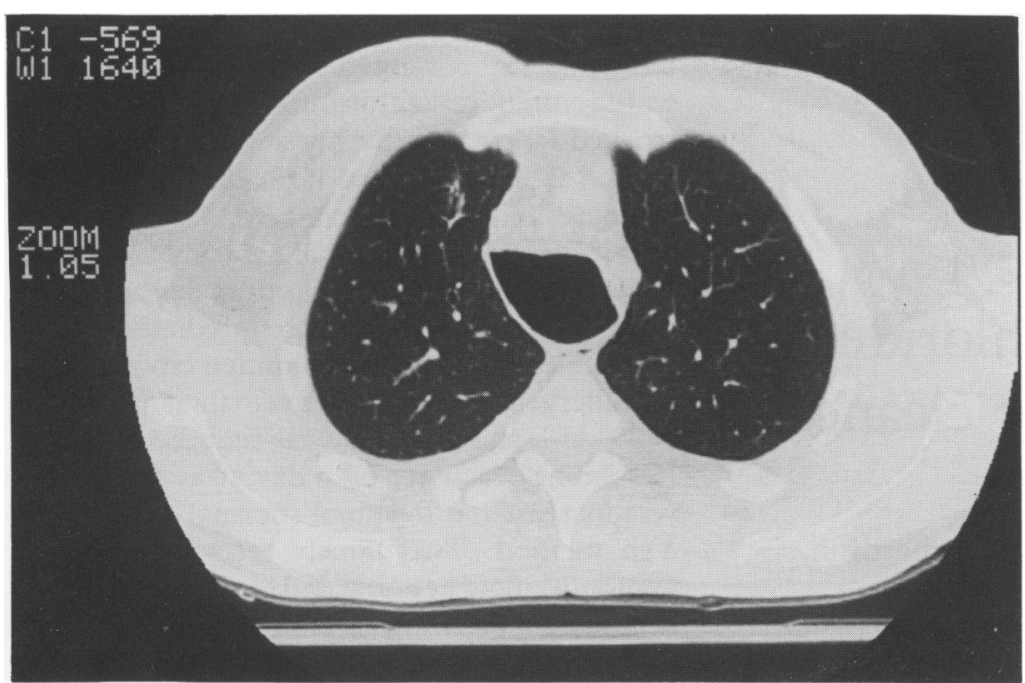

Figure 2 Computed tomographic appearances of tracheobronchomegaly showing considerable dilatation of trachea with unusual cross sectional profile. Surrounding lung parenchyma of the upper lobes shows no evidence of bronchiectasis.

airway obstruction, spirometric values were normal, and exercise tolerance had returned to the premorbid state.

\section{Discussion}

Although first recognised at post-mortem examination by Czyhlarz in $1897^{1}$ and latterly described by Mounier-Kuhn in $1932,{ }^{2}$ the term tracheobronchomegaly derives from the review of Katz et al in $1962 .{ }^{3}$ This congenital disorder, principally of the upper airways, remains a rare cause of recurrent lower respiratory tract infection. It is characterised by abnormal dilatation of the trachea and major bronchi. Clinical manifestations range from the asymptomatic individual diagnosed by screening ${ }^{4}$ to those with recurrent lower respiratory tract infection and bronchiectasis, in some cases dating from childhood. ${ }^{5}$ Most cases occur in men who present in middle age, ${ }^{6}$ although an autosomal recessive pattern of inheritance has been suggested from studies in one family. ${ }^{4}$ Histological studies support the concept of a congenital defect or atrophy of the elastic and smooth muscle tissue of the trachea and main bronchi. Diagnosis depends on the demonstration of the characteristically large trachea and proximal bronchi by plain radiography or bronchography, or more recently by computer aided tomography ${ }^{8}$ or magnetic resonance imaging. ${ }^{9}$ A mild degree of tracheal dilatation may be caused by diffuse pulmonary fibrosis but it seems unlikely that this represents the same disease. ${ }^{10}$ The diagnosis can be easily overlooked on plain radiography; although the tracheal silhouette was noted to be abnormal in our patient its significance was not appreciated until computed tomographic scanning revealed the diagnosis. Another interesting feature of our patient was the severity of the presenting illness, bearing in mind the paucity of symptoms before presentation. This may have been partly caused by the administration of corticosteroids.

We are reminded to consider this rare cause of recurrent lower respiratory tract infection in patients presenting with pulmonary sepsis, and to use corticosteroids with caution in patients with apparent chronic airflow limitation.

1 Czyhlarz ER. Ueber ein pulsiondivertikel der trachea mit bemerkungen ueber das verhaltn der elattichen fasern an normalentracheen und bronchiem. Zentralbl Allg Pathol 1897;18:721-8.

2 Mounier-Kuhn P. Dilatation de la trachee: constatations radiographiques et bronchoscopiques. Lyon Med 1932; 150:106.

3 Katz L, Levine M, Herman P. Tracheobronchomegaly: the Mounier-Kuhn syndrome. $A \mathcal{F}$ 1962;88:1084-94.

4 Johnston RF, Green RE. Tracheobronchiomegaly: report of five cases and demonstration of familial occurrence. $A m$ Rev Respir Dis 1965;91:35-50.

5 Rahbar M, Tabatabai DJ. Tracheobronchomegaly. Br $\mathcal{F}$ Dis Chest 1971;65:65-8.

6 Boomsma JD, Schraufnagel DE. A man with a large trachea. Chest 1991;100:809-11.

7 Van Schoor J, Joos G, Pauwels R. Tracheobronchomegaly the Mounier Kuhn syndrome: report of two cases and a review of the literature. Eur Respir $\mathcal{F}$ 1991;4:1303-6.

8 Shin MS, Jackson RM, Ho KJ. Tracheobronchomegaly (Mounier-Kuhn syndrome): CT diagnosis. $A \mathscr{R}$ 1988; 150:777-9.

9 Rindsberg S, Friedman AC, Fiel SB, Radecki PD. MRI of tracheobronchomegaly. Can Assoc Radiol f 1987;38:126-8.

10 Schraufnagel DE, Boomsma JD. Tracheobronchomegaly. Chest 1992;102:1307. 